\title{
CONSTRUCTION AND CHARACTERIZATION OF GALOIS ALGEBRAS WITH GIVEN GALOIS GROUP
}

\author{
TADASI NAKAYAMA
}

Recently H. Hasse ${ }^{\text {) }}$ has given an interesting theory of Galois algebras, which generalizes the well known theory of Kummer fields; an algebra $\mathfrak{A}$ over a field $\Omega$ is called a Galois algebra with Galois group $G$ when $\mathfrak{U}$ possesses $G$ as a group of automorphisms and $\mathfrak{I}$ is $(G, \Omega)$-operator-isomorphic to the group ring $G(\Omega)$ of $G$ over $\Omega .^{2}$ ) On assuming that the characteristic of $\Omega$ does not divide the order of $G$ and that absolutely irreducible representations of $G$ lie in $\Omega$, Hasse constructs certain $\mathcal{Q}$-basis of $\mathfrak{A}$, called factor basis, in accord with Wedderburn decomposition of the group ring and shows that a characterization of $\mathfrak{I}$ is given by a certain matrix factor system which defines the multiplication between different paris of the factor basis belonging to different characters of $G$. Now the present work is to free the theory from the restriction on the characteristic. We can indeed embrace the case of non-semisimple modular group ring $G(\Omega)$.

1. Decomposition of group ring. ${ }^{3)} \quad$ Let $G$ be a finite group whose absolutely irreducible representations lie in a field $\Omega$. Let $G=G(\Omega)$ be its group ring over 2. Let

$$
1=\sum_{\kappa=1}^{k} \sum_{i=1}^{f(\kappa)} e_{i}^{(\kappa)}
$$

be a decomposition of 1 into a sum of mutually orthogonal primitive idempotent elements in $\$$, where the left-(or, right-)ideals generated by $e_{1}^{(\kappa)}, \ldots, e_{f(\kappa)}^{(\kappa)}$ are isomorphic while those generated by $e_{i}^{(\kappa)}, e_{j}^{(\lambda)}$ with $\kappa \neq \lambda$ are not. Let $c_{i j}^{(\kappa)}$ be, for each $k$, a corresponding system of matric units. For simplicity's sake we denote $e_{1}^{(\kappa)}$ by $e^{(\kappa)}$. Let

Received December 26, 1949 ; revised March 5, 1950. The revision was to make the paper into a form of abstract, so as to transfer the original full account, with further supplement, to Crelles Journal according to Prof. Hasse's kind invitation.

1) $[2]$.

$\Rightarrow$ Hasse demands further that $\mathfrak{A}$ be associative, commutative and, moreover, semisimple.

3) Cf. e.g. [3]. 


$$
\mathfrak{v}^{(\kappa)}=\left(\begin{array}{c}
e^{(\kappa)} \\
t_{2}^{(\kappa)} \\
\vdots \\
t_{i(\kappa)}^{(\kappa)}
\end{array}\right)
$$

be an (independent) $\Omega$-basis of the right ideal $e^{(\kappa / S}$ taken in accord with a composition series. We have, for $z \in \mathbb{G}$,

$$
\mathfrak{v}^{(\kappa)} z=V^{(\kappa)}(z) \mathfrak{b}^{(\kappa)}
$$

with a representation $V^{(\kappa)}$ of $\mathscr{B}$ in $\Omega$. We assume that $e^{(1)}$ corresponds to the 1-representation of $G$. We can, and shall, take $\sum_{z \in G} z$ for $t_{v(1)}^{(1)}$.

As for the left-ideal $\left(\mathcal{S} e^{(\kappa)}\right.$ we take its basis

$$
\mathfrak{u}^{(\kappa)}=\left(e^{(\kappa)}, s_{2}^{(\kappa)}, \ldots, s_{v(\kappa)}^{(\kappa)}\right)
$$

in the following more specified manner. Let namely the $q$-th residue-module in a composition series of. $\left(S e^{(\kappa)}\right.$ correspond to $e^{(\kappa q(\kappa))}$ (i.e. be isomorphic to $\left(\mathfrak{S} e^{\left(\kappa_{q}(\kappa)\right) / M} e^{\left(\kappa \kappa_{q}(\kappa)\right)}\right.$, where $\mathfrak{N}$ denotes the radical of $(\mathbb{S})$, and take a generator $r_{q}^{(\kappa)}(\in \mathbb{S})$ of the residue-module; $r_{q}^{(\kappa)}$ may be taken from $\left.e^{\left(\kappa \kappa_{q}(\kappa)\right.}\right)\left(S e^{(\kappa)}\right.$, and we really employ $e^{(\kappa)}$ as $r_{1}^{(\kappa)}$. Then

$$
\left(\left(e^{(\kappa)}, c_{21}^{(\kappa)}, \ldots\right), \quad\left(e^{(\kappa, 2(\kappa))}, c_{21}^{(\kappa)(\kappa))}, \ldots\right) r_{2}^{(\kappa)}, \ldots \ldots\right)
$$

forms a basis of $\left(\mathfrak{S} e^{(\kappa)}\right.$, which we take for $\mathfrak{H}^{(\kappa)}$ in (4).

Now we introduce a matrix

$$
\mathfrak{T}^{(\kappa)}=\left(\mathfrak{b}^{(\kappa)}, s_{2}^{(\kappa)} \mathfrak{b}^{(\kappa)}, \ldots, s_{v(\kappa)}^{(\kappa)} \mathfrak{b}^{(\kappa)}\right)
$$

in $(\mathscr{S}$; it is the transpose of the Kronecker product, so to speak, of the transposes of $\mathfrak{u}^{(\kappa)}, \mathfrak{y}^{(\kappa)}$. Denote the matrix consisting of the first $f(\kappa)$ columns of $\mathfrak{I}^{(\kappa)}$ by $\mathfrak{V}^{(\boldsymbol{k})}$, i.e.

$$
\mathfrak{B}^{(\kappa)}=\left(\mathfrak{b}^{(\kappa)}, c_{21}^{(\kappa)} \mathfrak{b}^{(\kappa)}, \ldots, c_{f(\kappa) !}^{(\kappa)} \mathfrak{b}^{(\kappa)}\right)
$$

We have

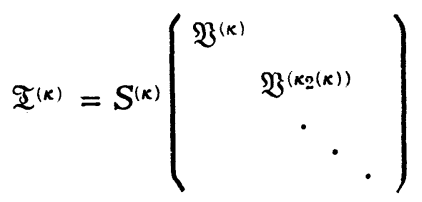

with a matrix $S^{(\kappa)}$ in $\Omega$. Here

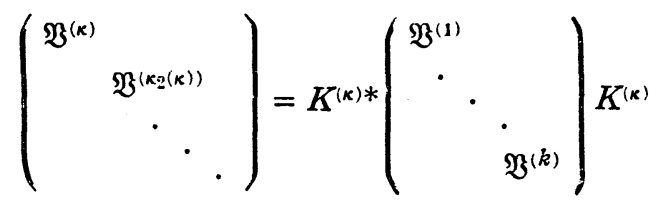

with matrices $K^{(\kappa)}, K^{(\kappa) *}$ possessing one 1 in each column or row respectively. Thus 


$$
\mathfrak{I}^{(\kappa)}=S^{(\kappa)} K^{(\kappa) *}\left(\begin{array}{lll}
\mathfrak{V}^{(1)} & & \\
\cdot & & \\
& \cdot & \\
& \cdot \\
& \mathfrak{V}^{(k)}
\end{array}\right) K^{(\kappa)} \cdot
$$

If $\mathfrak{x}$ is any column of elements of $\mathbb{B}$ satisfying $\mathfrak{d} z=V^{(\kappa)}(z) \mathfrak{x}$, then there exists an element $x$ in $\mathscr{B}$ such that $\mathfrak{x}=x \mathfrak{D}^{(\kappa)}$. Hence

$$
\mathfrak{x}=\mathfrak{T}^{(\kappa)} X
$$

with a column $X$ in $\Omega$; in fact $X$ is the first column of the matrix corresponding to $x$ in the representation of $\mathbb{G}$ defined by $\mathscr{C} e^{\kappa}$ with respect to our basis $\mathfrak{u}^{(\kappa}$.

Now, Kronecker products of $V^{i k}$ are decomposed, directly, into certain numbers of $V^{(\kappa)}$.) Thus

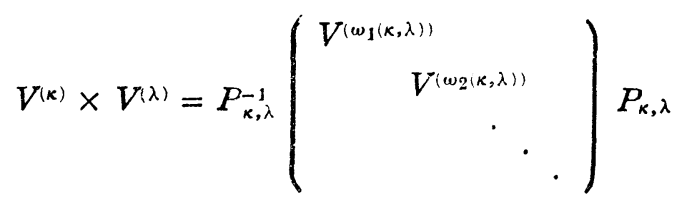

with a non-singular matrix $P_{\kappa, \lambda}$ in $\Omega$. There is a matrix $G_{\kappa, \lambda}$ possessing one 1 in each column such that

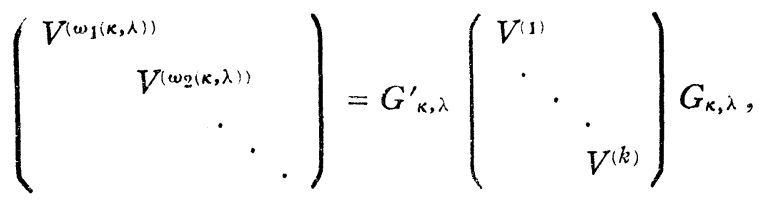

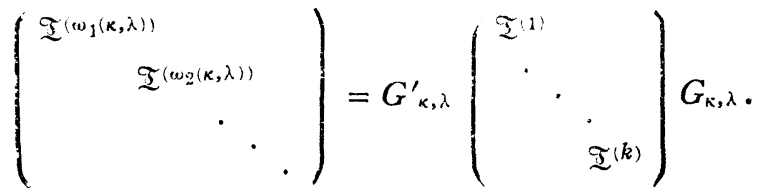

We have next

$$
V^{(\lambda)} \times V^{(\kappa)}=J_{\kappa, \lambda}^{-1}\left(V^{(\kappa)} \times V^{(\lambda)}\right) J_{\kappa, \lambda}
$$

with permutation matrix $J_{\kappa, \lambda}$. Further we may assume

$$
\omega_{i}(\lambda, \kappa)=\omega_{i}(\kappa, \lambda), \quad G_{\lambda, \kappa}=G_{\kappa, \lambda} \text { and } P_{\lambda, \kappa}=P_{\kappa, \lambda} J_{\kappa, \lambda} .
$$

Finally we quote the following particular case of the Nesbitt-Brauer-Nakayama orthogonality relation ${ }^{51}$

$$
\sum_{z \in G} V^{(1)}(z)=\left(\begin{array}{ll}
0 & \gamma \\
0 & 0
\end{array}\right) \quad(\gamma \neq 0), \quad \sum_{z \in G} V^{(\kappa)}(z)=0 \quad(\kappa \neq 1) .
$$

2. Galois algebra. Let $\mathfrak{A}$ be an algebra, not necessarily associative, over $\Omega$

4) See [5].

5) See [1], [4]. 
which has $G$ as a group of automorphisms. We call $\mathfrak{A}$ a Galois algebra, with Galois group $G$, when the right $\mathbb{S}(=G(\Omega))$-module $\mathfrak{A}$ is isomorphic to $\mathbb{B}$ itself (i.e. when $\mathfrak{A}$ possesses a normal basis). Let, with such a Galois algebra $\mathfrak{A}, \quad \sim$ denote an isomorphism of $\mathbb{B}$ and $\mathfrak{A}$. We have

$$
\tilde{\mathfrak{S}}^{(\kappa)} z=V^{(\kappa)}(z) \widetilde{\mathfrak{B}}^{(\kappa)}
$$

for $z \in \mathfrak{S}$. So $\left(\tilde{\mathfrak{B}}^{(\kappa)} \times \tilde{\mathfrak{B}}^{(\lambda)}\right) z=\left(V^{(\kappa)} \times V^{(\lambda)}\right)(z)\left(\tilde{\mathfrak{B}}^{(\kappa)} \times \widetilde{\mathfrak{B}}^{(\lambda)}\right)$, or

$$
P_{\kappa, \lambda}\left(\tilde{\mathfrak{B}}^{(\kappa)} \times \tilde{\mathfrak{B}}^{(\lambda)}\right) z=\left(\begin{array}{r}
V^{\left(\omega_{1}(\kappa, \lambda)\right)}(z) \\
V^{\left(\omega_{2}(\kappa, \lambda)\right.}(z) \\
\cdot \\
\cdot
\end{array}\right) P_{\kappa, \lambda}\left(\widetilde{\mathfrak{B}}^{(\kappa)} \times \tilde{\mathfrak{B}}^{(\lambda)}\right) .
$$

Hence, from an observation in 1 (and the isomorphism property of $\sim$ ),

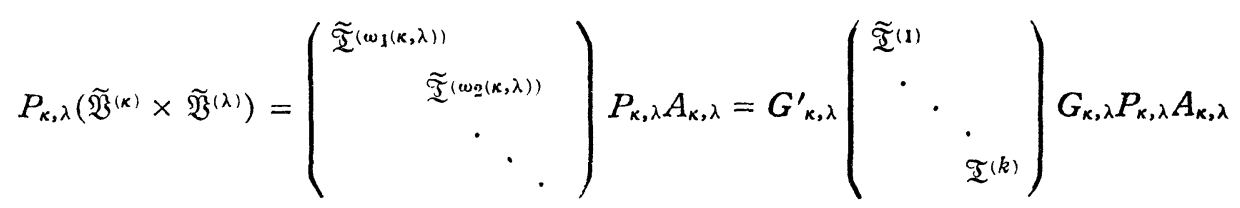

i.e.

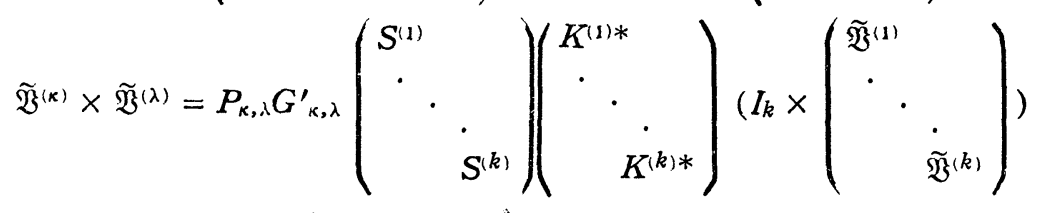

$$
\left(\begin{array}{cc}
K^{(1)} & \\
\cdot & \\
& \cdot \\
& K^{(k)}
\end{array}\right) G_{\kappa, \lambda} P_{\kappa, \lambda} A_{\kappa, \lambda}
$$

with uniquely determined matrix $A_{\kappa, \lambda}$ of type $(v(\kappa) v(\lambda), f(\kappa) f(\lambda))$ in $\Omega, I_{k}$ being unit matrix of degree $k$. Taking $A_{\kappa, \lambda}$ for each pair $(\kappa, \lambda)$ we obtain a system $\left\{A_{\kappa, \lambda} ; \kappa, \lambda=1,2, \ldots, k\right\}$ of matrices in $\Omega$.

Conversely any system $\left\{A_{\kappa, \lambda}\right\}$, with each $A_{\kappa, \lambda}$ possessing type $(v(\kappa) v(\lambda)$, $f(\kappa) f(\lambda)$ ), defines a Galois algebra with Galois group $G$. Namely, if we introduce $g=\sum f(\kappa) v(\kappa)$ elements, arrange them into $k$ matrices $\tilde{\mathfrak{B}}^{(\kappa)}$ of respective type $(v(\kappa), f(\lambda))$, define by virtue of (17) an $\Omega$-linear multiplication in the $\Omega$-module $\mathfrak{U}$ spanned by the elements, considered as being independent, and set (16), then we see that $\mathfrak{i f}$ becomes a Galois algebra with Galois group $G$ corresponding to the given system $\left\{A_{\kappa, \lambda}\right\}$.

Now, similar consideration can be made for $\widetilde{\mathfrak{T}}^{(\kappa)} \times \tilde{\mathfrak{I}}^{(\lambda)}$ too, to give

$$
\tilde{\mathfrak{T}}(\kappa) \times \widetilde{\mathfrak{I}}^{(\lambda)}=P_{\kappa, \lambda} G^{\prime}{ }_{\kappa, \lambda}\left(\begin{array}{cc}
\widetilde{\mathfrak{I}}^{(1)} & \\
\cdot & \\
& \cdot \\
& \dot{\tilde{\mathfrak{I}}^{(k)}}
\end{array}\right) G_{\kappa, \lambda} P_{\kappa, \lambda} B_{\kappa, \lambda}
$$


with again uniquely determined matrix $B_{\kappa, \lambda}$, of degree $v(\kappa) v(\lambda) ; A_{\kappa, \lambda}$ is composed of certain $f(\kappa) f(\lambda)$ columns of $B_{\kappa, \lambda}$. Also the system $\left\{B_{\kappa, \lambda}\right\}$ characterizes $\mathfrak{A}$, but it must be observed that it can not, in general, be taken arbitrarily, contrary to $\left\{A_{\kappa, \lambda}\right\}$. Indeed, elements of $B_{\kappa, \lambda}$ can be expressed linearly by those of $A$ 's, the expression depending on $G$ (and $\Omega$ ) only (but not on $\mathfrak{A}$ ), which we write in

$$
B_{\kappa, \lambda}=B_{\kappa, \lambda}\left(\left\{A_{\kappa, \lambda}\right\}\right) \text {. }
$$

Let, with our same $\mathfrak{A}$, a second ( $\mathfrak{B}$-) isomorphism of $\mathbb{S}$ and $\mathfrak{A}$ be denoted by - There exists a regular element $a$ in $\mathbb{S}$ such that $\bar{x}=\widetilde{a x} \quad(x \in \mathbb{S})$. We see that

$$
P_{\kappa, \lambda}^{-1} G_{\kappa, \lambda}^{\prime}\left(\begin{array}{ccc}
U^{(1)}(a)^{-1} & & \\
\cdot & \cdot & \\
& \cdot & \\
& & U^{(k)}(a)^{-1}
\end{array}\right) G_{\kappa, \lambda} P_{\kappa, \lambda} B_{\kappa, \lambda}\left(U^{(\kappa)}(a) \times U^{(\lambda)}(a)\right)
$$

plays for $\overline{\mathfrak{I}}^{(\kappa)}$ the roll of $B_{\kappa, \lambda}$ for $\tilde{\mathfrak{I}}^{(\kappa)}$, where $U^{(\kappa)}$ denotes the representation defined by the basis $\mathfrak{l l}^{(\kappa)}$ of $B e^{(\kappa)}$. Thus:

(Under the assumption that all absolutely irreducible representations of $G$ lie in $\Omega$ ) to each Galois algebra $\mathfrak{A}$ over $\Omega$ with Galois group $G$ is associated a class of systems $\left\{A_{\kappa}, \lambda\right\}$ muiually related by transformation which takes $A_{\kappa, \lambda}$ into the matrix consisting of the 1 st $, \ldots, f(\lambda)-t h, v(\lambda)+1 s t, \ldots, v(\lambda)+f(\lambda)-t h, \ldots$ columns of (20) with $B_{\kappa, \lambda}=B_{\kappa, \lambda}\left(\left\{A_{\kappa, \lambda}\right\}\right)$, a being regular element in (5). Multiplication in $\mathfrak{A}$ is given in terms of $\left\{A_{\kappa}, \lambda\right\}$ by (17), and operation of $G$ on $\mathfrak{i}$ by (16). Conversely, any system $\left\{A_{\kappa, \lambda}\right\}$ of matrices in $\Omega$, with respective type $(v(\kappa) v(\lambda)$, $f(\kappa) f(\lambda))$, gives rise to a Galois algebra over $\Omega$ with Galois group $G$.

3. Associativity, commutativity and semisimplicity. If $\mathfrak{A}$ is commutative, then the permutation matrix $J_{\kappa, \lambda}$ in (13) gives also $J_{\kappa, \lambda}^{-1}\left(\widetilde{\mathfrak{I}}^{(\kappa)} \times \widetilde{\mathfrak{I}}^{(\lambda)}\right) J_{\kappa, \lambda}=\widetilde{\mathfrak{I}}^{(\lambda)}$ $\times \widetilde{\mathfrak{I}}^{(\kappa)}$. We have then

$$
B_{\lambda, \kappa}=J_{\kappa, \lambda}^{-1} B_{\kappa, \lambda} J_{\kappa, \lambda},
$$

which gives in fact necessary and sufficient condition for the commutativity of $\mathfrak{A}$; if we take (19) (and perhaps its trivial inverse) into account, the condition can be regarded as being in terms of $\left\{A_{\kappa}, \lambda\right\}$.

On considering $V^{(\kappa)} \times V^{(\lambda)} \times V^{(\mu)}$, let next $H_{\kappa, \lambda, \mu}$ and $L_{\kappa, \lambda, \mu}$ be permutation matrices satisfying

$$
\begin{gathered}
H_{\kappa, \lambda, \mu}^{-1}\left(\begin{array}{c}
V^{(\kappa)} \times V^{\left(\omega_{1}(\lambda, \mu)\right)} \\
. \\
.
\end{array}\right) H_{\kappa, \lambda, \mu}=V^{(\kappa)} \times\left(\begin{array}{c}
V^{\left(\omega_{1}(\lambda, \mu)\right)} \cdot \\
.
\end{array}\right), \\
L_{\kappa, \lambda, \mu}^{-1}\left(\begin{array}{c}
V^{\left(\omega_{1}\left(\omega_{\xi}(\kappa, \lambda), \mu\right)\right)} \\
\cdot .
\end{array}\right) L_{\kappa, \lambda, \mu}=\left(\begin{array}{c}
V^{\left(\omega_{1}\left(\kappa, \omega_{1}(\lambda, \mu)\right)\right)} \\
. \\
.
\end{array}\right) .
\end{gathered}
$$


Then

(24) $L_{\kappa, \lambda, \mu}\left(\begin{array}{c}P_{\kappa, \omega_{1}(\lambda, \mu)} \\ \cdot \\ \cdot\end{array}\right) H_{\kappa, \lambda, \mu}\left(I_{v(\kappa)} \times P_{\lambda, \mu}\right)=Q_{\kappa, \lambda, \mu}\left(\begin{array}{c}P_{\omega_{I}(\kappa, \lambda), \mu} \\ \cdot \\ \cdot\end{array}\right)\left(P_{\kappa, \lambda} \times I_{v(\mu)}\right)$ with a non-singular matrix $Q_{\kappa, \lambda, \mu}$ commutative with the representation $\left(\begin{array}{c}V^{\left(\omega_{1}\left(\omega_{1}(\kappa, \lambda), \mu\right)\right.} \cdot \\ \cdot\end{array}\right)$. There is matrix $R_{\kappa, \lambda, \mu}$ such as

$$
Q_{\kappa, \lambda, \mu}^{-1}\left(\begin{array}{c}
\mathfrak{T}^{\left(\omega_{1}\left(\omega_{1}(\kappa, \lambda) \mu\right)\right.} \\
\cdot .
\end{array}\right)=\left(\begin{array}{c}
\mathfrak{T}^{\left(\omega_{1}\left(\omega_{1}(\kappa, \lambda) \mu\right)\right.} \\
\cdot . \\
\cdot
\end{array}\right) R_{\kappa, \lambda, \mu} .
$$

These matrices $H_{\kappa, \lambda, \mu}, L_{\kappa, \lambda, \mu}, Q_{\kappa, \lambda, \mu}$ and $R_{\kappa, \lambda, \mu}$ are all determined by $G$ (and $\Omega$ ) only. Calculating $\left(\widetilde{\mathfrak{T}}^{(\kappa)} \times \widetilde{\mathfrak{T}}^{(\lambda)}\right) \times \widetilde{\mathfrak{T}}^{(\mu)}$ and $\widetilde{\mathfrak{T}}^{(\kappa)} \times\left(\tilde{\mathfrak{T}}^{(\lambda)} \times \widetilde{\mathfrak{I}}^{(\mu)}\right)$, we find that

$$
\begin{aligned}
& \left(\begin{array}{c}
P_{\omega_{1}(\kappa, \lambda), \mu}^{-1} \\
\cdot
\end{array}\right)\left(\begin{array}{c}
B_{\omega_{1}(\kappa, \lambda), \mu} \\
\cdot \\
\cdot
\end{array}\right)\left(P_{\kappa, \lambda} \times I_{v(\mu)}\right)\left(B_{\kappa, \lambda} \times I_{v(\mu)}\right) \\
= & R_{\kappa, \lambda, \mu} L_{\kappa, \lambda, \mu}\left(\begin{array}{c}
P_{\kappa, \omega_{i}(\lambda, \mu)} \\
\cdot \\
\cdot
\end{array}\right)\left(\begin{array}{c}
B_{\kappa, \omega_{1}(\lambda, \mu)} \\
\cdot \\
\cdot
\end{array}\right) H_{\kappa, \lambda, \mu}\left(I_{v(\kappa)} \times P_{\lambda, \mu}\right)\left(I_{v(\kappa)} \times B_{\lambda, \mu}\right)
\end{aligned}
$$

is necessary and sufficient for the associativity of $\mathfrak{A}$; the condition may be seen again as being in terms of $\left\{A_{\kappa}, \lambda\right\}$.

Finally, since the system $\left\{A_{\kappa, \lambda}\right\}$ gives, by (17), the multiplication table of $\mathfrak{A}$, the regular discriminant of $\mathfrak{U}$ can be expressed by means of $\left\{A_{\kappa, \lambda}\right\}$. Provided $\mathfrak{A}$ is associative (that is, (26) holds) its non-vanishing is necessary and sufficient in order that $\mathfrak{i}$ be absolutely semisimple and its capacities be all indivisible by the characteristic of $\Omega$. However, on assuming both the commutativity and associativity we can obtain a second expression for the discriminat (whose nonvanishing is now necessary and sufficient for the absolute semisimplicity of $\mathfrak{A}$ ) as follows. Namely, the trace of an element of $\mathfrak{A}$ may then be given as the sum of its transforms by $G$. So the matrix composed of the traces of elements of $\tilde{\mathfrak{V}}^{(\kappa)} \times \tilde{\mathfrak{V}}^{(\lambda)}$ is given by

$$
\sum_{z \in G}\left(\tilde{\mathfrak{V}}^{(\kappa)} \times \tilde{\mathfrak{V}}^{(\lambda)}\right) z=\sum_{z}\left(P_{\kappa, \lambda}^{-1}\left(\begin{array}{c}
\tilde{\tilde{\mathfrak{I}}^{(}\left(\omega_{1}(\kappa, \lambda)\right)} \\
\cdot \\
\cdot
\end{array}\right) P_{\kappa, \lambda} A_{\kappa, \lambda}\right) z .
$$

Making use of the orthogonality relation (15) we find that this is equal to

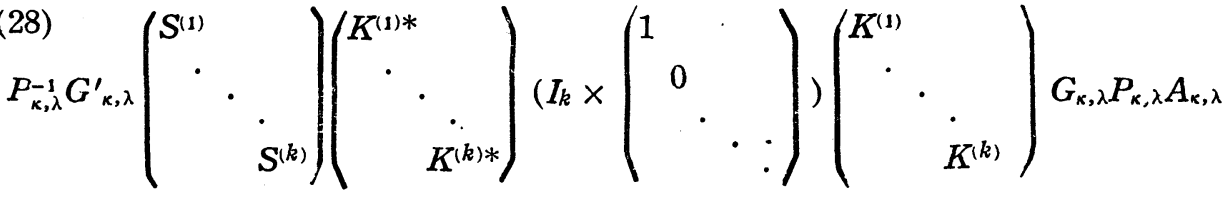

multiplied by $r$ and the trace of $\tilde{1}$ (which is not 0 ). Now the part of our dis- 
criminant matrix corresponding to the products of the basis elements from $\widetilde{\mathfrak{V}}^{\langle\kappa\rangle}$ and $\widetilde{\mathfrak{B}}^{(\lambda)}$ can be obtained from $(28)$ by virtue of a certain, easily describable rearrangement of elements (except a non-zero scalar factor independent of $\kappa, \lambda$ ).

These together offer criterion for the associativity, commutativity and absolute semisimplicity of the Galois algebra $\mathfrak{U}$ given by $\left\{A_{\kappa, \lambda}\right\}$.

\section{REFERENCES}

[1] R. Brauer, Hypercomplex arithmetic and a theorem of Speiser, Com. Mat. Helvetici (1945).

[2] H. Hasse, Invariante Kennzeichnung Galoisscher Körper mit vorgegebener Galoisgruppe, forthcoming in Crelles Journal.

[3] T. Nakayama, Some studies on regular representations, induced representations and modular representations, Ann. Math. 39 (1938).

[4] C. Nesbitt-R. Thrall, Some ring theorems with applications to modular representations, Ann. Math. 47 (1946).

[5] M. Osima, Note on the Kronecker product of representations of a group, Proc. Acad. Japan 17 (1941).

Nagoya University 